\title{
Venise entre Orient et Occident
}

Venice between East and West

\section{Michèle Vignaux}

\section{(2) OpenEdition \\ Journals}

\section{Édition électronique}

URL : http://journals.openedition.org/shakespeare/1497

DOI : 10.4000/shakespeare.1497

ISSN : 2271-6424

Éditeur

Société Française Shakespeare

Édition imprimée

Date de publication : 13 décembre 2009

Pagination : 1-15

ISBN : 2-9521475-6-6

Référence électronique

Michèle Vignaux, «Venise entre Orient et Occident », Actes des congrès de la Société française

Shakespeare [En ligne], 27 | 2009, mis en ligne le 13 décembre 2009, consulté le 06 mai 2019. URL :

http://journals.openedition.org/shakespeare/1497; DOI : 10.4000/shakespeare.1497 


\title{
Shakespeare et l'Orient
}

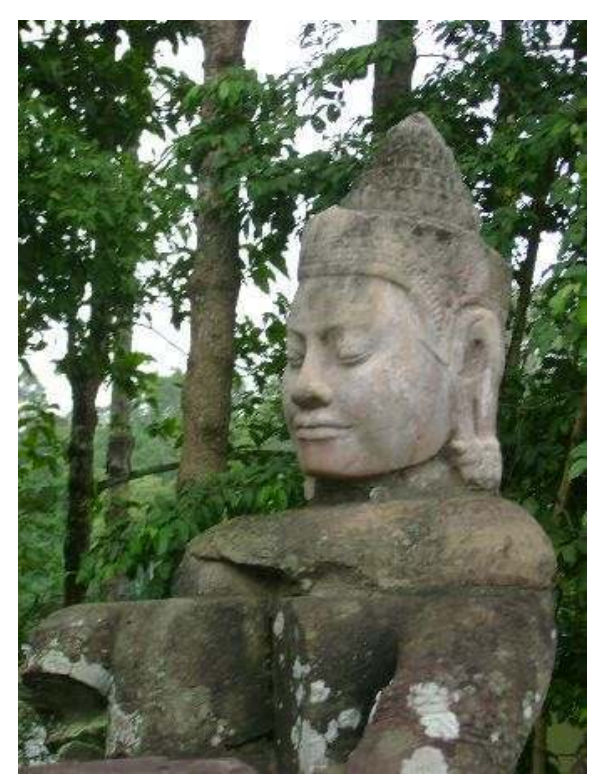

\author{
actes du Congrès \\ organisé par la \\ les 12, 13 et 14 mars 2009 \\ textes réunis par \\ Pierre KAPITANIAK \\ sous la direction de \\ Jean-Michel DÉPRATS
}

SOCIÉTÉ FRANÇAISE SHAKESPEARE 
COUVERTURE :

photo J.-M. Déprats 2008

conception graphique et logo

Pierre Kapitaniak

\section{(C) 2009 Société Française Shakespeare \\ Institut du Monde Anglophone \\ Université de Paris III - Sorbonne Nouvelle \\ 5 rue de l'École de Médecine 75006 Paris \\ www.societefrancaiseshakespeare.org}




\title{
VENISE ENTRE ORIENT ET OCCIDENT
}

\author{
Michèle VignauX
}

Venise est la seule ville dont le nom figure explicitement dans le titre de deux pièces de Shakespeare: The Merchant of Venice et Othello, the Moor of Venice. D'où vient cet intérêt particulier pour cette ville et, par delà un décor au moins partiellement commun, quel(s) lien(s) le nom de Venise tisse-t-il entre ces deux pièces ? Cette question est ici envisagée sous l'angle de la conversion religieuse, dans cette ville carrefour réputée tolérante envers les étrangers de toutes confessions, où Shakespeare met en scène deux juifs et un Maure, convertis au christianisme avec des succès variables. Ce faisant, il explore des questions qui touchaient l'Angleterre de fort près, qu'il s'agît de la fracture, interne au christianisme, entre catholiques et protestants, ou des relations entre chrétiens et non chrétiens. L'étude des divers types de conversion - centripète ou centrifuge, spontanée ou contrainte, réelle ou apparente, masculine ou féminine - fait apparaître les liens entre conversion et identité et conduit à une conclusion en forme de double aporie, en ceci que la conversion au christianisme, tout en étant indispensable, se révèle le plus souvent impossible ou, en cas de conversion réussie, se voit reprocher de conduire à un brouillage et donc à une menace pour les identités.

Venice between East and West Venice is the only city whose name appears in the title of two of Shakespeare's plays : The Merchant of Venice and Othello, the Moor of Venice. What can account for such an interest in this particular city? What kind of links does the name of Venice establish between these two plays, beyond their (partly) common setting ? These questions are approached from the angle of religious conversion, in that city which had a reputation for tolerance towards foreigners of all kinds of confessions, where Shakespeare has staged two Jews and a Moor who are converted to Christianity with various fortunes. In so doing, he touches upon issues which were not unrelated with the situation in England, whether it be the internal divide between Catholics and Protestants, or the relations between Christians and non Christians. The study of the various types of conversion (centripetal vs centrifugal, voluntary vs compulsory, sincere vs apparent, masculine vs feminine) reveals the links that exist between conversion and identity, and leads to the aporetic conclusion that conversion to Christianity is at once necessary and generally impossible to achieve fully or, when successful, accused of blurring identities.

$\mathrm{V}$

enise est la seule ville dont le nom figure explicitement dans le titre de deux pièces de Shakespeare: The Merchant of Venice et Othello, the Moor of Venice. Pourquoi cette ville, dont ni Shakespeare ni la plupart de ses contemporains n'avaient une connaissance directe, et qui pourtant semble avoir exercé sur eux une certaine fascination ${ }^{1}$ ? La question est l'objet d'un débat déjà ancien, récemment renouvelé par la déconstruction de ce que David McPherson a appelé le «mythe de Venise ${ }^{2}$ », mythe au demeurant largement entretenu par les Vénitiens eux-mêmes. L’un des aspects du

${ }^{1}$ J'emprunte le terme «fascination » à Anne Geoffroy, dont on consultera avec profit la thèse "Genèse d'une fascination. Les représentations culturelles de Venise dans l'Angleterre de la première modernité (1549-1642) ", soutenue en 2008 à l'Université de Versailles-St Quentin. Voir également l'article de Christophe Camard, "L'Italie selon Shakespeare et Ben Jonson: l'altérité dans un théâtre sans décor », LISA VI.3, 2008.

2 David C. McPherson, Shakespeare, Jonson, and the Myth of Venice, Newark, U. of Delaware P., 1990. 
débat qui nous intéresse plus particulièrement ici porte sur le degré d’identification entre la Sérénissime et la capitale de l'Angleterre. Si l'idée d'une identification stricte de Venise et de Londres est désormais abandonnée, il reste que les deux métropoles partageaient un certain nombre de traits susceptibles de retenir l'attention du dramaturge. On s'attachera ici à une caractéristique commune aux deux villes : leur situation de port et de carrefour maritime, qui fait d'elles tout à la fois des lieux d'échanges et de préjugés. Plus précisément, on s'interrogera sur le double mouvement simultané d'ouverture à l'autre et de repli sur soi caractéristique de cette ère de découvertes, double mouvement qui peut se lire comme la conséquence d'une position perçue de plus en plus comme ambivalente, de la part de populations à l'identité encore mal définie, oscillant entre esprit conquérant (à l'ouest) et crainte d'être détruites (par l'est) ${ }^{3}$. Cette situation, bien résumée par Thomas More dans son Dialogue of Comfort Against Tribulation de 1534, s'était accentuée un demi-siècle plus tard ${ }^{4}$, pour s'aggraver encore au XVII ${ }^{e}$ siècle, dominé par un sentiment de fatalité selon lequel le christianisme, après avoir supplanté le judaïsme, était lui-même voué à être supplanté par l'Islam ${ }^{5}$. Comme le fait remarquer à juste titre Nabil Matar, les juifs ayant été chassés en 1290, l'« autre »par excellence, pour les Anglais du début de l'époque moderne, était moins l'Indien du Nouveau monde que le musulman de l'Empire ottoman (et plus

3 Cf. Daniel J. Vitkus, Turning Turk: English Theater and the Multicultural Mediterranean, 1570-1630, New York, Palgrave Macmillan, 2003 : « when alien figures are presented on the early modern stage, they partake of both xenophobic and xenophilic tendencies in English culture » et, un peu plus loin, «English anxieties about cultural pollution, miscegenation, or religious conversion were intense, but at the same time the cultural, ethic, and religious differences were often embraced and internalized as English culture began to absorb and articulate those differences as a part of its own process of selfidentification. » (p. 22-23). Voir également Matthew Dimmock, New Turkes. Dramatizing Islam and The Ottomans in Early Modern England, Aldershot \& Darlington, Ashgate Publishing, 2005, chap. 1, et Ania Loomba, Shakespeare, Race, and Colonialism, Oxford, O.U.P., 2002, p. 13.

4 Cf. Nabil I. Matar, Turks, Moors, and Englishmen in the Age of Discovery, New York, Columbia U. P., 1999, xi-268 p., p. 9 ainsi que, du même auteur, Islam in Britain, 15581685, Cambridge, C.U.P., 1998, p. 12-14.

5 Cf. N. Matar, "The Renegade in English Seventeenth-Century Imagination », Studies in English Literature 33, 1993, p. 502 et Daniel J. Vitkus, "Early Modern Orientalism : Representations of Islam in Sixteenth- and Seventeenth-century Europe », in David R. Blanks et Michael Frassetto, éds., Western Views of Islam in Medieval and Early Modern Europe Perception of Other, New York, Macmillan, 1999, p. 208. 
généralement du pourtour méditerranéen), figure la plus répandue de populations non chrétiennes sur le sol anglais ${ }^{6}$.

Il n'est guère possible cependant de considérer les relations avec l'Orient de façon isolée, comme Matar le reconnaît lui-même en établissant un lien entre l'Orient et le Nouveau monde, selon un modèle triangulaire d'après lequel les Britanniques, faute de pouvoir dominer l'Orient comme ils avaient commencé de conquérir le Nouveau monde, auraient transféré le discours sur les Indiens d'Amérique aux populations orientales (Turcs et Maures), de manière en quelque sorte incantatoire relevant de la pensée magique, mais qui se serait finalement révélée avoir eu une valeur proleptique et performative. En outre, on ne peut qu'être frappé par la proximité de certaines formulations décrivant les contacts entre populations anglaise et « autres » - qu'il s'agisse d'Écossais, d'Irlandais, d'Indiens d'Amérique, ou de « Turcs » et de « Maures » - et notamment par une sorte de parallèle entre la crainte de « turning Turk » et celle de « going native ", toutes deux renvoyant à une menace pour l'intégrité identitaire $^{8}$. Au demeurant, le vocabulaire utilisé pour désigner les

${ }^{6}$ N. Matar, Turks, Moors, and Englishmen, p. 3-4. Il n'est que de comparer les douzaines de pièces mettant en scène des Turcs et des Maures durant l'âge d'or du théâtre anglais (des années 1580 aux années 1630) à l'absence de pièce mettant en scène les Indiens d'Amérique. Pour ce qui est des juifs, Lionel Ifrah rappelle que l'Angleterre fut le premier pays d'Europe à expulser ses juifs, avant d'accueillir quelques marranes d'Espagne et du Portugal à la fin du Xve siècle. Il mentionne également l'existence d'une petite communauté de juifs d'origine vénitienne, composée de musiciens et de luthiers, appelés au début des années 1540 à la cour d'Henri viII (De Shylock à Samson: Juifs et judaïsme en Angleterre au temps de Shakespeare et Milton, Paris, Champion, 1999, p. 13-16).

7 N. Matar, Turks, Moors, and Englishmen, p. 15-18, ainsi que Islam in Britain, p. 11-13. En d'autres termes, la rhétorique impériale et l' «idée » d'empire auraient constitué un discours pré-orientaliste (au sens qu'Edward Said donne à ce terme dans son ouvrage Orientalism, Londres, Routledge \& Kegan Paul, 1978) qui aurait ouvert la voie à la domination effective des XVIII ${ }^{\mathrm{e}}$ et XIX ${ }^{\mathrm{e}}$ siècles. Il convient cependant d'user de la plus grande prudence dans l'extrapolation des travaux de Said à la période précédant le XVIII ${ }^{\mathrm{e}}$ siècle. En effet, même s'il est vrai que le discours orientaliste est antérieur à l'invasion de l'Égypte par Bonaparte en 1798, il faut se garder de l'écueil d'une lecture téléologique rétrospective dans la mesure où, comme le souligne Vitkus, «the orientalist discourse described by Edward Said [...] began to emerge in an era when the European relationship to the Orient was not yet one of colonial dominance, but one of anxiety and awe on the part of Europeans. [...] it was largely a response to the cultural superiority of the Muslims, particularly those of alAndalus. » ("Early Modern Orientalism... », in Blanks and Frassetto, eds., Western Views of Islam, p. 209-10). L'espace méditerranéen des XVI et XVII ${ }^{\mathrm{e}}$ siècles est en fait un espace complexe, qui échappe largement aux oppositions binaires Orient/Occident ou colonisateur/colonisé (cf. D. Vitkus, Turning Turk, p. 3 sq).

${ }^{8}$ Sur ce dernier aspect, voir Michael Neill, « Broken English and Broken Irish : Nation, Language, and the Optic of Power in Shakespeare's Histories ", Shakespeare Quarterly 45 (1994) p. 7, 9-10 et 14, n. 51, ainsi que Vitkus, Turning Turk, p. 9 et Ania Loomba, Shakespeare, Race and Colonialism, p. 18. 
populations de couleur était notoirement instable, ce qui conduisait à une sorte d'amalgame de tous les non blancs, non chrétiens ${ }^{9}-$ ou, si différence on faisait, c'était au moyen de stéréotypes opposant par exemple le Turc cruel et tyrannique au Maure naïf et superstitieux, ou encore lascif, en proie à des émotions incontrôlables, et prompt à la vengeance $^{10}$.

En conséquence, plutôt qu'une opposition bipolaire Orient/Occident, ou même qu'un modèle triangulaire AngleterreOrient-Nouveau monde, un modèle de cercles concentriques organisés autour de l'Angleterre est sans doute mieux à même de rendre compte des relations avec l'Orient dans toute leur complexité : un premier cercle intègrerait les marges de l'Angleterre (Pays de Galles et Écosse) dans ce qui devait devenir la Grande-Bretagne ${ }^{11}$, un deuxième inclurait l'Irlande, un troisième intègrerait l'Europe continentale, et enfin un quatrième l'Orient proche (Empire ottoman et Afrique du nord) et le Nouveau monde ${ }^{12}$. Ce modèle doit être encore compliqué pour tenir compte de la division interne à la chrétienté consécutive à la Réforme, et de ses répercussions jusque dans le Nouveau monde, à travers les entreprises colonisatrices de l'Angleterre et de l'Espagne notamment ${ }^{13}$.

Cette division interne à la chrétienté généra également, à partir des années 1530, un usage purement rhétorique du mot "Turk», utilisé en un sens métaphorique, comme une insulte que catholiques et protestants se jetaient mutuellement à la face, l'expression «new turkes » renvoyant, selon les auteurs, aux luthériens et autres « hérétiques », ou aux catholiques ou «papistes », chaque parti accusant en outre l'autre d'être la cause, par sa conduite indigne ou en encourageant les ferments de division, de la menace que les Turcs

\footnotetext{
${ }^{9}$ Voir G.K. Hunter, « Elizabethans and Foreigners », Shakespeare Survey 17, 1964, p. 40 et 51 ainsi que Ania Loomba, Shakespeare, Race and Colonialism, p. 46, 54, 67 et 71.

${ }^{10} \mathrm{Cf}$. Matar, Turks, Moors, and Englishmen, p. 13.

${ }^{11} \mathrm{Cf}$. les planches sur les Pictes à la fin de l'ouvrage de Thomas Harriot, A Briefe and True Report of the Newfoundland of Virginia, dans l'édition de 1590, New York, Dover Publications, 1972, xv-91 p., p. 75-85.

12 Nous laisserons de côté l'Afrique sub-saharienne, perçue avant tout comme un réservoir de main d'œuvre servile et qui ne constituait donc pas de menace, ainsi que l'Orient plus lointain, encore largement méconnu.

13 Cf. John H. Elliott, Empires of the Atlantic World, Britain and Spain in America 14921830, New Haven, Yale U.P., 2006, xx-546 p. L'opposition entre les pratiques colonisatrices de l'Angleterre protestante et de l'Espagne catholique constitue un véritable leitmotiv du récit de Sir Walter Ralegh, The Discoverie of the Large, Rich and Bewtiful Empyre of Guiana, 1595, éd. Neil L. Whitehead, Manchester U.P., 1997, viii-232 p.
} 
faisaient planer sur la chrétienté ${ }^{14}$. La dichotomie entre pays catholiques et pays protestants ne se limitait d'ailleurs pas à des échanges sur le plan polémique, elle engendrait également des jeux d'alliances complexes et fluctuants avec l'Orient musulman, caractérisés par une hésitation constante entre unité pan-chrétienne et rivalités intra-européennes ${ }^{15}$.

À partir de la fin des années 1570, dans le sillage de l'excommunication d'Élizabeth et durant la décennie suivante, marquée par les complots successifs au nom de Marie Stuart jusqu'à son exécution en 1587 et par l'affrontement avec l'Armada espagnole en 1588, l'Angleterre, profitant des besoins en armement de l'Empire ottoman alors engagé dans une longue guerre contre la Perse (de 1578 à 1590), et espérant pouvoir bénéficier ultérieurement de l'alliance turque contre l'Espagne, développa sa politique de coopération et d'échanges commerciaux avec l'Empire ottoman avec la signature de Capitulations en 1580 et la création de la Compagnie du Levant l'année suivante. Cette politique n'est pas sans évoquer celle de Venise, petite principauté qui, à partir de sa situation géographique « charnière » et à la faveur d'une brèche entre l'Empire des Habsbourg et l'Empire ottoman, avait réussi à se tailler un véritable empire maritime dont la prospérité dépendait d'une adroite politique diplomatique avec les Ottomans dans laquelle le pragmatisme des intérêts commerciaux l'emportait sur les considérations idéologiques. C'est ainsi que son retrait de la Sainte Ligue en 1573 avait permis à Venise de marquer son indépendance envers la papauté et d'obtenir grâce à un traité ce que la victoire de Lépante en 1571 ne lui avait pas accordé : un droit d'accès à Chypre, épisode suivi avec le plus grand intérêt par l'Angleterre ${ }^{16}$.

\footnotetext{
14 Cf. D. Vitkus, « Early Modern Orientalism »,p. 211-213 et M. Dimmock, qui précise : «In a strongly millenarian English culture the dominant tropes of "Catholic" and "Turke" were closely related as two complementary sides of the Antichrist, one who tyrannizes by "force", the other through "fraude" " (New Turkes, p. 91) à tel point que, selon Matar, "there is scarcely a mention of the Turks and Islam in early modern English eschatology without an immediate mention of the Pope and Catholicism» (Islam in Britain, p. 154). Voir également Dimmock, New Turkes, p. 57, 77-79, et 164.

15 Cf. D. Vitkus, «Early Modern Orientalism », p. 213-14 et " "Turning Turk" in Othello: The Conversion and Damnation of the Moor » Shakespeare Quarterly 48.2, 1997, p. 165

${ }^{16}$ Cf. William H. McNeill, Venice : The Hinge of Europe 1081-1797, Chicago, U of Chicago P, 1974, xvii-334 p., p. 140-41, ainsi que 183 sq. ; voir également Daniel Goffman, The Ottoman Empire and Early Modern Europe, Cambridge, C.U.P., 2002, xxiii-273 p., p. 139141 et 164 .
} 
Toutefois, si les alliances se concluaient et se renversaient avec une certaine versatilité, les représentations culturelles demeuraient beaucoup plus stables, et tandis que la perception du « Turc » devenait plus nuancée sous l'effet de contacts plus nombreux, plus pacifiques et mieux informés à travers des frontières plus perméables, attestés par tout un éventail de sources de plus en plus diversifiées (mémoires de captivité, dépositions de prisonniers, documents du Privy Council et autres documents officiels issus de contacts directs avec des musulmans) dont Matar souligne à juste titre l'importance ${ }^{17}$, les anciens clichés et stéréotypes hostiles hérités des traditions médiévales, tant historique que littéraire (romans médiévaux et légendes chevaleresques) subsistèrent jusque très avant dans le XVII siècle, véhiculés par toute une littérature qui n'hésitait pas pour cela à se tourner vers des épisodes historiques conflictuels, de sorte que l'idée que l'on se faisait du «Turc» s'en trouvait compliquée d'autant, couvrant toute une gamme de représentations où se côtoyaient les croyances populaires et l'érudition, les descriptions réalistes et le matériau merveilleux ou légendaire ${ }^{18}$.

Les années 1590 marquent un sommet dans l'alliance angloottomane, assorti d'un souci de maintenir une façade ostensiblement chrétienne en cette période où les Turcs, désormais sortis de leur longue guerre contre la Perse, pouvaient à nouveau se tourner contre la Pologne et la Hongrie ${ }^{19}$. Ce conflit plaçait l'Angleterre et Venise, partenaires commerciaux de la Turquie, dans une position de plus en plus délicate envers leurs coreligionnaires, ce qui allait conduire l'Angleterre à un changement de cap inéluctable dans les années 1600, amorcé dès la fin du règne d'Élisabeth et accentué sous Jacques Irer, dont l'accession en 1603 fut marquée par la réédition, à l'occasion de

17 Turks, Moors, and Englishmen, p. 7. Voir également Rosalyn L. Knutson, « Elizabethan Documents, Captivity Narratives, and the Market for Foreign History Plays », English Literary Renaissance 26, 1996.

18 Cf. Dimmock, New Turkes, p. 16 et 85-86; David Blanks, "Western Views of Islam in the Premodern Period ", in David Blanks et M. Frassetto, eds., Western Views of Islam, p. 23, 35 ainsi que, dans le même volume, D. Vitkus, « Early Modern Orientalism », p. 209.

${ }^{19}$ Shakespeare fait une brève allusion à ce conflit, qui devait durer de 1593 à 1606, dans une autre pièce de la période, située à Vienne : Measure for Measure (1604), au début de I.ii. Sur ce conflit et ses conséquences diplomatiques, voir M. Dimmock, New Turkes, (p. 166-171) pour ce qui concerne l'Angleterre et Daniel Vitkus, " "Turning Turk" in Othello », p. 162-63 pour ce qui concerne Venise. 
son couronnement, de son poème épique de 1585 : Lepanto $^{20}$, à la gloire de la victoire remportée par les flottes coalisées de la Sainte Ligue contre les Turcs à Lépante. L'hostilité persistante envers les Turcs, qualifiés par Richard Knolles dans sa Generall Historie of the Turks (1603) de "scourge of God and present terror of the world ", devait se prolonger tout au long du XVII ${ }^{\mathrm{e}}$ siècle, y compris après la défaite des Turcs aux portes de Vienne en 1683 et après le traité de Karlowitz (1699) qui marquait le déclin militaire de l'Empire ottoman et la fin de toute menace, en une hostilité de principe passablement figée envers les musulmans et l'Islam dans le seul champ resté libre pour l'affrontement : celui de la polémique doctrinale ${ }^{21}$.

Bien que The Merchant of Venice (1596-97) et Othello relèvent, par leur période de composition, de contextes politiques différents et que l'une mette en scène un juif, l'autre un Maure, la reprise du Merchant of Venice, au côté d'Othello (1603-04), au cours de la saison théâtrale de 1604-05 autorise, voire encourage, les rapprochements entre les deux pièces - au point qu'on a pu envisager Othello en termes de réécriture du Merchant of Venice. Dans un article très riche auquel il est impossible de rendre justice dans le cadre de cette étude, Julia Reinhard Lupton fonde son rapprochement sur deux marqueurs de l'altérité : l'exogamie manifeste et une structure typologique opposant le christianisme, selon le cas, au judaïsme ou à l'Islam ${ }^{22}$. Pour Maurice Hunt, le « paradigme vénitien » chez Shakespeare réside dans « certain dynamic relationships between a persecutory Christian culture and a potentially savage alien - a Turk, a Moor, or a Jew — who exists both

\footnotetext{
${ }^{20}$ La Sainte Ligue, constituée sous l'égide de la papauté entre le roi d'Espagne Philippe II, Venise, la plupart des États italiens et les chevaliers de Malte, devient sous la plume de Jacques I $^{\text {er }}$ un symbole de l'unité pan-chrétienne. Sur ce poème, voir Emrys Jones, «Othello, Lepanto, and the Cyprus Wars », Shakespeare Survey 21, 1968, qui signale quelques variantes entre les deux éditions, notamment l'addition des expressions « faithles and circumcised Turbaned Turkes » et « the baptiz'd race ».

${ }^{21} \mathrm{Cf}$. Matar, Islam in Britain, p. 185-86 et 189-90.

22 Julia Reinhard Lupton, «Othello Circumcised : Shakespeare and the Pauline Discourse of Nations ", Representations 57, 1997, p. 75 : " Othello [...] has often been read as a rewriting of The Merchant of Venice: both are set in the mercantile city-state of Venice, both employ clearly marked "others," and both use the theme of conspicuous exogamy to heighten the conventional comedic situation of young lovers blocked by an old father. Merchant exhibits a comedic structure sharply typological in its countering of Jewish justice and Christian mercy, a set of scriptural coordinates more carefully submerged yet all the more powerfully at work in Othello as well» et Lupton conclut : " [In Shakespeare's Venetian plays, the] Christian-humanist discourse always operates as a universalism minus the circumcised [Jews and Muslims] » (p. 84).
} 
without and within the city ${ }^{23} »$. Nous voudrions suggérer ici une autre piste, et envisager les deux pièces sous l'angle de la conversion, question centrale dans l'Europe de la première modernité et dans les pièces vénitiennes de Shakespeare, en liaison avec la situation géographique de cette ville d'entre-deux, étape intermédiaire où les marranes espagnols et portugais optaient définitivement pour le christianisme ou retournaient au judaïsme ${ }^{24}$.

Il convient d'emblée de distinguer deux grandes catégories de conversion, que l'on peut qualifier respectivement de " centripète » et de « centrifuge ». La première, la seule à être dotée de connotations positives, était principalement le fait de juifs convertis au christianisme, tandis que la seconde, d'ailleurs plutôt désignée par le terme clairement négatif d'apostasie, était principalement le fait de chrétiens renégats, et s'effectuait au profit de l'Islam. Les deux pièces vénitiennes de Shakespeare mettent en scène les forces centripètes, avec trois conversions au christianisme, dont deux à partir du judaïsme dans Le Marchand de Venise : celles de Jessica et de Shylock; quant à Othello, sauf à considérer qu'il est né chrétien (ce qui, sans être tout à fait exclu, reste très improbable), la question de savoir si sa conversion au christianisme s'est effectuée à partir du paganisme ou de l'Islam reste largement indécidable ${ }^{25}$ et d'ailleurs relativement indifférente. Pour autant, les forces centrifuges ne sont pas absentes, plutôt tenues à distance, au moins jusqu'à un certain point. Ainsi, la conversion d'Othello, antérieure au début de la pièce, se révèle fragile, instable, et aboutit finalement à la rechute tragique dans l'«erreur»; dans la tragi-comédie The Merchant of Venice, la conversion spontanée de Jessica relève de la romance, tandis que celle de Shylock, beaucoup plus problématique, est repoussée à la fin de la pièce, ce qui exclut toute possibilité de remise en cause, sans pourtant que soit dissipé le

\footnotetext{
23 Maurice Hunt, «Shakespeare's Venetian paradigm : Stereotyping and sadism in The Merchant of Venice and Othello ", Papers on Language and Literature, Spring 2003, p. 1, http://findarticles.com/p/articles/mi_qa3708/is_200304/ai_n9225201 (consulté le 12 février 2009).

${ }^{24}$ Cf. Brian Pullan, The Jews of Europe and the Inquisition of Venice, 1550-1670, Oxford, Basil Blackwell, 1983, p. xiii-xiv.

25 Lupton ( Othello Circumcised », p. 78), suggère que la première partie, qui relève de la comédie romantique, ou encore de ce que Susan Snyder a appelé la "matrice comique» (The Comic Matrix of Shakespeare's Tragedies : Romeo and Juliet, Hamlet, Othello, and King Lear, Princeton, N.J., Princeton U.P., 1979), évoque une conversion à partir du paganisme et qu'ensuite, Othello est de plus en plus associé à l'idée du musulman converti au christianisme qui retombe dans l'« erreur » de l'Islam.
} 
profond malaise qu'elle suscite, en particulier chez les spectateurs modernes. La question de la conversion se révèle éminemment complexe du fait de la multiplicité des aspects à prendre en compte: caractère spontané ou contraint de la conversion, avec d'inévitables répercussions sur sa sincérité; enjeux liés au genre, selon que l'on a affaire à une conversion masculine ou féminine ; articulation entre nature et culture et lien avec la définition de l'identité enfin - toutes questions que nous allons examiner tour à tour.

Historiquement, la question de la sincérité de la conversion se posa d'abord et principalement à propos des juifs au moment de la reconquête de l'Espagne musulmane en 1492. Malgré une assimilation massive des juifs convertis tout au long du $\mathrm{Xv}^{\mathrm{e}}$ siècle, beaucoup restaient suspects de continuer à pratiquer un judaïsme clandestin. D'autant que l'Inquisition, instaurée dès 1480 dans le but de sonder les consciences afin de démasquer ceux dont la conversion n'était que conformité extérieure, avait rapidement révélé ses limites et son échec à distinguer véritablement les juifs des chrétiens par la foi, dans la mesure où elle reposait sur les déclarations des intéressés, toujours sujettes à caution ${ }^{26}$. Le soupçon de crypto-judaïsme était également alimenté par le fait que ceux qui, parmi ces "nouveaux chrétiens ", comme on les appelait aussi, avaient bravé l'interdiction d'émigration pour gagner des pays musulmans (principalement des territoires ottomans) où ils avaient trouvé une plus grande tolérance - ou indifférence - étaient dans leur grande majorité retournés au judaïsme ${ }^{27}$.

${ }^{26}$ Cf. Jerome Friedman, "Jewish Conversion, the Spanish Pure Blood Laws and Reformation: A Revisionist View of Racial and Religious Antisemitism », Sixteenth Century Journal XVIII.1, 1987, p. 15 : «Alleged crypto-Jewish Marranos were unlike earlier heretics [...they] would deceitfully affirm any and all tenets of orthodox faith and anything else required of them. It was assumed Marranos would say the expedient thing and hence Inquisition authorities dismissed statements of faith in Christianity as meaningless. ".

27 Contrairement à ce qui se passait en Espagne, où ce retour à la foi originelle était passible de mort et faisait courir des risques à l'individu mais aussi à la communauté juive qui le réadmettait en son sein (cf. J. Friedman, op. cit., p. 13), Bernard Lewis explique : « In Muslim lands [...] apostasy from Christianity to Judaism was a matter of indifference. Muslim jurists in any case often took the view that a forced conversion was not valid and that renouncing such a conversion did not constitute apostasy. » (Cultures in Conflict: Christians, Muslims, and Jews in the Age of Discovery, Oxford, O.U.P., 1995, 101 p., p. 38) Au surplus, la conversion était également tenue en suspicion par les autorités chrétiennes et rabbiniques, promptes à utiliser le mythe de la conversion purement extérieure pour renforcer leur position au sein de leurs communautés respectives, ce qui plaçait de nombreux convertis dans une situation des plus délicates de rejet de la part des deux communautés (J. Friedman, op. cit., p. 14-15 et 20-21). 
En Angleterre où, entre autres pays protestants du nord de l'Europe, une communauté de marranes portugais avaient trouvé refuge, le soupçon de crypto-judaïsme et les préjugés antisémites étaient également prêts à resurgir à la première occasion, comme en témoigne le procès du Dr Lopez à Londres en 1594. Mais les résonances avec la situation anglaise ne se limitaient pas à la situation des juifs, et on ne peut s'empêcher d'effectuer certains rapprochements avec la question catholique. Malgré la volonté proclamée de la reine Élizabeth de ne pas sonder le secret des âmes (« not making windows into men's souls ») et de se contenter d'une conformité extérieure de pratique, dans le contexte d'anticatholicisme national, particulièrement aigu durant toute la période qui s'étend de la Rébellion du nord de 1569 aux années qui suivirent la conspiration des poudres de 1605, la question de la religion était indissociable de celle de la loyauté à la reine et à la nation. On peut entendre un écho de la situation de nombreux catholiques dans le « divided duty » évoqué par Desdémone lorsqu'elle met en lumière le conflit entre son devoir filial et son devoir d'épouse $(\text { I.iii.180 })^{28}$, qui à son tour ne peut manquer d'évoquer la pratique d'équivocation mise au point par les jésuites comme riposte à la fameuse «bloody question ${ }^{29}$ ».

Aux craintes suscitées par le crypto-judaïsme et le catholicisme s'ajoutait le spectre de la conversion (forcée ou volontaire) à l'Islam. Cette crainte ancienne, déjà présente dans nombre de récits relatifs aux croisades, se trouvait ravivée par l'accroissement du trafic maritime et de la piraterie, et les récits de captivité qui accompagnaient la collecte d'aumônes destinées à payer la rançon de chrétiens faits prisonniers par des navires turcs et soumis à la tentation de la conversion à l'Islam pour échapper à la servitude avaient rendu cette menace familière aux Élisabéthains ${ }^{30}$. Ceux qui résistaient étaient célébrés comme des héros

\footnotetext{
28 William Shakespeare, Othello, the Moor of Venice, éd. Michael Neill, coll. World's Classics, Oxford, O.U.P., 2006.

29 Je me permets de renvoyer aux pages que j'ai consacrées à ces questions dans L'Invention de la responsabilité, Paris, Presses de l'École Normale Supérieure, 1995, 352 p., p. 99-107. Friedman signale l'importance du rôle des «nouveaux chrétiens » dans la fondation de la Compagnie de Jésus (art. cité, p. 23). Voir également sur le contexte d'anticatholicisme national, Carol Z. Wiener, "The Beleaguered Isle. A Study of Elizabethan and Early Jacobean Anti-Catholicism ", Past and Present 51, 1971, p. 27-29 et sur le lien entre foi religieuse et allégeance nationale, Peter Berek, "The Jew as Renaissance Man », Renaissance Quarterly 51, 1998, p. 129.

$3^{\circ}$ Cf. Rosalyn L. Knutson, «Elizabethan Documents, Captivity Narratives, and the Market for Foreign History Plays », English Literary Renaissance 26, 1996.
} 
de la «vraie » foi ; ceux qui avaient succombé à la tentation de la conversion étaient l'objet de tous les efforts pour tenter de les ramener dans le giron de la religion chrétienne, par l'édification ou par la peur. Mais c'était sans compter la force d'attraction exercée par l'Empire ottoman à travers les récits des exploits de pirates renégats, tel le célèbre Barberousse, ou par l'exemple de chrétiens qui y avaient atteint des situations sans commune mesure avec ce à quoi ils auraient pu prétendre dans leur pays d'origine ${ }^{31}$. Face à ces obstacles opposés par la réalité, les contemporains se tournèrent vers les ressources de la fiction, particulièrement au théâtre, avec deux pièces emblématiques : A Christian Turn'd Turke, or The Tragicall Lives and Deaths of the two Famous Pyrates, Ward and Dansiker (1609-10) de Robert Daborne et The Renegado, or the Gentleman of Venice (1623-24) de Philip Massinger, les dramaturges renchérissant volontiers sur l'horreur morale dépeinte par les théologiens en fabriquant «sur mesure » un destin tragique pour les renégats non repentis, qui devinrent une nouvelle incarnation du mal dans une galerie déjà bien fournie ${ }^{32}$.

La conversion à l'Islam était fréquemment décrite en termes érotiques sur le mode de la tentation, associée à la faiblesse d'une volonté dominée par des appétits incontrôlés 33 . Ania Loomba fait état de l'existence de toute une tradition littéraire remontant au Moyen Âge, et mettant en scène la séduction d'un chrétien par une musulmane qui le prend au piège de ses charmes pour l'attirer vers une foi licencieuse et le conduire à sa perte, comme la forme de transgression sexuelle la plus courante. Le héros échappe généralement à son destin funeste grâce à la mort de la séductrice ou à sa propre conversion au christianisme, prélude au mariage avec le héros ${ }^{34}$. Autre intrigue

${ }^{31}$ Voir par exemple D. Vitkus, « Early Modern Orientalism », in D. Blanks et M. Frassetto, eds., op. cit., p. 215-16, ainsi que N. Matar, "The Renegade ", p. 489-91, et Islam in Britain, p. 20 et p. 50-51.

$3^{2}$ Cf. N. Matar, Islam in Britain, p. 52 : « The renegade of drama was thus as much an invention of the English imagination as the Machiavellian malefactor, the Faustian atheist or the Moor. He was a type representing the new villain in England's conflict with the antiChrist: as the Machiavellian villain embodied all that Protestant Englishmen feared in Catholicism ; as the Faustian atheist represented the Mephistophelians of the new science; as the Moor represented all that was "oriental" and alien to England, so did the renegade represent the internal evil that threatened Christendom. »

33 Cf. D. Vitkus, "Turning Turk" in Othello: The Conversion and Damnation of the Moor » Shakespeare Quarterly 48.2, 1997, p. 145-46 et 153-57.

34 Ania Loomba, «"Delicious Traffick" : Alterity and Exchange on Early Modern Stages », Shakespeare Survey 52, 1999, p. 211. 
classique, qui constitue en quelque sorte le pendant « romantique » de la précédente: une princesse musulmane tombe amoureuse d'un chevalier chrétien, se convertit au christianisme et l'épouse, tandis que le Sultan son père est tué ou se convertit lui aussi 35 .

Il convient de s'arrêter sur la dissymétrie des traitements réservés aux hommes et aux femmes. Sans surprise, dans la réalité comme dans la fiction, les châtiments infligés aux femmes en cas de transgression de linterdit touchant aux relations sexuelles et au mariage en dehors de la communauté religieuse étaient toujours plus sévères que ceux qui étaient appliqués aux hommes - et ce, dans les trois religions monothéistes ${ }^{36}$. Moins prévisible, en revanche, est l'accueil qui était réservé à l'un et à l'autre sexe par la communauté d'adoption, où la dissymétrie jouait au contraire en faveur des femmes, dont la conversion religieuse coïncide généralement avec un mariage, tandis qu'un homme converti au christianisme était voué au célibat ou à la destruction ${ }^{37}$. La logique sous-jacente à cette dissymétrie de traitement était vraisemblablement liée au fait que, chez les chrétiens comme chez les musulmans, la religion était transmise par le père et que, du moins aux yeux des chrétiens, la conversion étant toujours suspecte d'instabilité, il importait que la foi du père demeurât inchangée ${ }^{3}$. Le lien entre filiation et transmission de la religion conduit à la question des liens entre conversion et identité ou, pour le dire autrement, à la question de savoir ce qui, dans l'identité, relevait de la nature et ce qui relevait de la culture.

Devant l'échec de l'Inquisition à distinguer véritablement les juifs des chrétiens par la foi, l'Espagne se dota à partir de 1530 d'un outil biologico-juridique, sous la forme des lois de pureté du sang, faisant appel à des critères plus « objectifs » que les déclarations des intéressés. Ces lois constituent un tournant radical dans l'histoire de l'idée de race en ceci qu'elles faisaient de la foi religieuse non plus un

35 Cf. D. Vitkus, « Early Modern Orientalism », in D. Blanks et M. Frassetto, éds., op. cit., p. 216.

36 Cf. A. Loomba, "Delicious Traffick" ", p. 210 : "According to Islamic jurisprudence, Muslim men could marry Christian or Jewish women [...] but Muslim women could not marry non Muslim men without risking a death penalty ».

37 Cf. D. Vitkus, « Early Modern Orientalism », in D. Blanks et M. Frassetto, éds., op. cit., p. $209,216$.

$3^{8}$ Il en va différemment dans le judaïsme traditionnel, où la religion est transmise par la mère, ce qui apparente le mariage de Jessica avec Lorenzo à une trahison de sa foi d'origine ; du point de vue chrétien en revanche, en l'absence de conversions masculines au judaïsme, la question ne se pose guère durant la période. 
choix culturel, mais un donné biologique ; avec pour conséquence que le sang juif, "dégénéré ", était considéré comme inaccessible au baptême et à la grâce, ce qui n'allait évidemment pas sans poser de problème, notamment à propos de Jésus, qui était juif, tout comme saint Paul, le plus célèbre des convertis ${ }^{39}$ !

De 1550 à 1670 , la république de Venise posséda elle aussi son Inquisition, moins connue que son homologue espagnole mais tout aussi centrale pour notre propos, et riche d'enseignements quant à la conception de l'identité en liaison avec la conversion religieuse. Brian Pullan a étudié dans le détail les diverses catégories de juifs qui eurent maille à partir avec l'Inquisition et leur devenir. Il ressort de son étude que les marranes espagnols et portugais qui gagnaient le ghetto ${ }^{40}$ pour y mener une vie discrète, respectueuse de l'ordre de la cité, n'étaient pas inquiétés: convertis sous la contrainte, ils se contentaient de retourner à leur foi d'origine, rejetant les faux-semblants et contribuant par là même à préserver la pureté du christianisme. Ceux qui, en revanche, refusaient de rejoindre le ghetto, ou encore les « nouveaux chrétiens» italiens, qui n'avaient pas l'excuse de la conversion sous la contrainte et retournaient au judaïsme, renversant la hiérarchie " naturelle » entre la religion dominante et la religion "vaincue », étaient perçus comme une menace non seulement pour l'Église, mais aussi pour l'ordre civil et la stabilité sociale. Une dernière catégorie regroupait les éternels indécis, ceux qui avaient le plus de risques d'être condamnés ${ }^{41}$.

On reconnaît la même logique à l'œuvre dans les verdicts de l'Inquisition vénitienne et dans l'établissement des lois de pureté du sang en Espagne : dans les deux cas, il s'agissait d'éliminer toute trace d'ambiguïté. Notons au passage que les mêmes observations s'appliquent aux renégats :

what was especially dangerous about the renegade was that he was no swarthy Moor or contorted Papist or necromancer but a common

39 Cf. J. Friedman, op. cit., p. 15, 18 et 27. Voir également A. Loomba, Shakespeare, Race and Colonialism, p. 39 et 68.

$4^{0}$ Rappelons que Venise, au xvI ${ }^{\mathrm{e}}$ siècle, fut la première ville à établir une séparation physique entre juifs et chrétiens, et à imposer aux juifs le port d'un signe distinctif (une rouelle de couleur jaune, la couleur infâmante de la folie et du crime).

${ }^{41}$ Brian Pullan, The Jews of Europe and the Inquisition of Venice, p. 315. 
English or Irish or Welsh man who wilfully renounced God and monarch and « turned Turke ${ }^{42} »$.

C'est tout le paradoxe des conversions réussies, qu'elles suscitent la nostalgie d'un temps où l'on savait à quoi s'en tenir, alors qu'un juif converti, un renégat brouillent les frontières de l'identité. C'est ce qui conduit Peter Berek à considérer le juif converti comme le paradigme de l'homme de la Renaissance, fasciné par les possibilités nouvelles de contribuer à façonner son identité et non plus de la subir comme un destin irrévocable ${ }^{43}$.

En réponse à la question liminaire: "pourquoi Venise? », je suggérerai que le choix de cette ville cosmopolite, où se côtoyaient chrétiens, juifs et Maures, permettait à Shakespeare d'aborder de manière détournée des questions dont nous avons vu qu'elles touchaient l'Angleterre de fort près, tout en lui offrant une liberté qu'il n'aurait pu avoir en situant ses pièces à Londres. On n'a pas manqué en effet de souligner la subtilité qui se manifeste dans ces pièces par rapport à bien des pièces du répertoire contemporain traitant de ces sujets éminemment complexes44. On se trouve en effet devant une aporie, en ce sens que la conversion est à la fois indispensable (le seul bon juif/musulman étant un converti au christianisme) et impossible, en raison d'une sorte de fatalité qui empêche la conversion véritable (c'est toute la tragédie d'Othello 45 ), ou au contraire parce que la conversion, trop parfaite, en vient à brouiller et à menacer les frontières des identités. À la dimension individuelle s'ajoutait en outre une dimension eschatologique, caractérisée par une tension constante entre le doute quant à la possibilité de la conversion et l'idée, largement répandue, que la conversion de tous les juifs constituait le

\footnotetext{
42 N. Matar, Islam in Britain, p. 52.

43 Peter Berek, « The Jew as Renaissance Man », Renaissance Quarterly 51 (1998), p. 129130 et 158.

44 Voir par exemple Ania Loomba, Shakespeare, Race and Colonialism : "Shakespeare's plays allow us to trace debates and contexts about race rather than only those opinions which became dominant as colonialism advanced. » (p. 44), ou encore "Othello problematizes the received equation of blackness with moral depravity that was being consolidated in Shakespeare's time by the newer ideology of race. » (p. 59).

45 Il n'est pas indifférent que, au moment de son suicide, Othello se désigne comme « the circumcisèd dog " (v.ii.354), en référence à cette marque commune aux juifs et aux musulmans dont J. R. Lupton fait observer qu'elle est à la fois indélébile et non génétique ( "Othello Circumcised», p. 75). Sur les derniers mots d'Othello, voir également M. Dimmock, New Turkes, p. 205-06 et D. Vitkus, «"Turning Turk" in Othello », p. 174-76.
} 
préalable nécessaire à la parousie et à l'avènement du royaume du Christ sur la terre ${ }^{46}$. Mais, comme le souligne Matar, l'Islam ne pouvait pas être intégré dans ce schéma téléologique, et par conséquent la vision d'un protestantisme triomphant héritée de John Foxe, si cruciale dans l'idéologie coloniale britannique en Amérique et en Afrique occidentale, ne pouvait s'appliquer dans les territoires musulmans du Levant. Ainsi, tandis que les juifs (réadmis en 1656, l'année suivant la Conférence de Whitehall) et les catholiques bénéficiaient progressivement d'une plus grande tolérance, et que les protestants du continent et les chrétiens d'Orient étaient accueillis comme réfugiés, les musulmans continuèrent de cristalliser l'hostilité bien au-delà du déclin militaire de l'Empire ottoman et de la fin de toute menace sur le front oriental de l'Europe ${ }^{47}$.

Michèle VIGNAUX Université Lumière Lyon 2

${ }^{46}$ Cf. M. Lindsay Kaplan, dans son introduction à William Shakespeare, The Merchant of Venice, éd. M. Lindsay Kaplan, coll. Texts and Contexts, Boston et New York, Bedford/St. Martin's, 2002, ix-377 p., p. 14

${ }^{47}$ N. Matar, Islam in Britain, p. 189-90. 